\title{
Remnant bone debris after ACL reconstruction surgery: a cause of heterotrophic calcification and patellar tendon ossification
}

\begin{abstract}
Patellar tendon ossification after anterior cruciate ligament reconstruction (ACLR) with Bone-patellar tendon-bone (BPTB) graft is an extremely rare complication. We are reporting a case of patellar tendon ossification and heterotrophic ossification after ACLR with BPTB graft in the same patient. This patient was managed non-operatively as it was not causing hindrance to his daily activity.
\end{abstract}

Keywords: patellar tendon ossification, heterotrophic ossification, BPTB, ACL
Volume 2 Issue 3 - 2018

\author{
Ravi Gupta,' Anil Kapoor, ${ }^{2}$ Ashwani Soni, ${ }^{3}$ \\ Gladson David Masih, ${ }^{4}$ Sourabh Khatri ${ }^{5}$ \\ 'Professor, Sports Injury Center, Government Medical College \\ Hospital, India \\ ${ }^{2}$ Senior Resident, Department of Orthopaedics, Government \\ Medical College Hospital, India \\ ${ }^{3}$ Assistant Professor, Department of Orthopaedics, Government \\ Medical College Hospital India \\ ${ }^{4}$ Senior Research Fellow, Department of Orthopaedics, \\ Government Medical College Hospital, India \\ ${ }^{5}$ Ex-postgraduate student, Department of Orthopaedics, \\ Government Medical College Hospital, India
}

Correspondence: Anil Kapoor, Senior Resident, Department of Orthopaedics, Government Medical College Hospital, India, Tel 8847364585, Email anil88gmch@gmail.com

Received: March 28, 2018 | Published: April 19, 2018

\section{Introduction}

ACLR is a commonly done procedure in orthopaedics. Hamstring tendon graft and BPTB graft are two most commonly used autograft for ACLR. BPTB graft is observed to be associated with more donor site morbidity as compared to hamstring tendon graft ${ }^{1}$. Patellar tendon ossification is one such rare complication seen with BPTB graft.

\section{Case presentation}

ACLR was done using BPTB graft in 30 years old badminton player (recreational). The time interval between index injury and surgery was 1 month. Patient also had a concomitant tear in the posterior horn of the medial meniscus for which meniscal repair was done. Next day after surgery knee bending (up to $90^{\circ}$ ), quadriceps strengthening exercises and non-weight bearing walking started. At 6 weeks, cycling, full knee bending and half squats were added to the rehabilitation program.

At 9 months patients presented to the clinic with anterior knee pain, restricted range of motion and difficulty in doing squats. On examination bony swelling was present on the medial aspect of the knee, tenderness was present at the donor site and the range of motion was restricted $\left(0-110^{\circ}\right)$. All stability was normal. The arthrometer $\left(\mathrm{KT}-1000^{\mathrm{TM}}\right)$ measurement showed that anterior translation of tibia was $4 \mathrm{~mm}$ in the normal knee and $5 \mathrm{~mm}$ in the operated knee. X-ray of the knee showed that patellar tendon ossification and heterotrophic calcification at the medial femoral condyle (Figure 1). Patient was managed non-operatively as he did not have any limitation of daily activity.

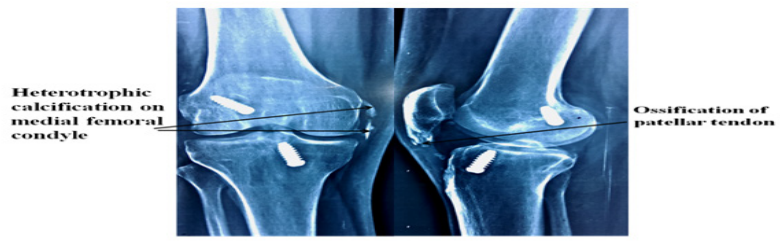

Figure I AP view of x-ray showing heterotrophic ossification on medial femoral condyle and lateral view of left knee $x$-ray showing patellar tendon ossification.

\section{Discussion}

BPTB graft is considered as a gold standard graft for ACLR because of better knee stabilty ${ }^{1,2}$ and higher return to sports after ACL reconstruction with BPTB graft..$^{1,3,4}$ However, BPTB graft is criticized for donor site morbidity like anterior knee pain, patellar fracture, quadriceps tendon rupture etc. ${ }^{5-7}$ Among the variously described complication of BPTB graft, patellar tendon ossification is one such rare complication. Patellar tendon ossification has been reported mainly in patients with knee injury, ${ }^{8}$ total knee replacement, ${ }^{9}$ partial patellectomy, ${ }^{10,11}$ intramedullary nail ${ }^{12}$ and after spinal cord injury. ${ }^{13}$ Patellar tendon ossification after ACLR is extremely rare. This complication has been reported by only few studies in literature ${ }^{14-18}$ (Table 1). The cause of patellar tendon ossification is not cleared. Gianluca Camillieri et al. ${ }^{16}$ gave hypothesis that persistence of bone debris inside the patellar tendon may trigger calcification. However, they did not have an immediate postoperative X-ray to prove their hypothesis. In the present case 
report, we had postoperative CT scan ( $5^{\text {th }}$ day) which showed the loose bone fragments in the joint (Figure 2) which support the hypothesis given by Gianluca Camillieri et al. ${ }^{16} \&$ Erdogan et al. ${ }^{19}$ gave another hypothesis he believed aggressive rehabilitation causes microtrauma within patellar tendon. ${ }^{19}$ However same rehabilitation protocol was followed by others patients and none of them showed patellar tendon ossification. Another possible pathogenesis given by Bonamo et al. ${ }^{20}$ he stated that devascularization of the graft during harvested results in avascular necrosis. Heterotrophic ossification is commonly encountered problem in orthopaedics. The incidence of heterotrophic ossification after ACL reconstruction with BPTB graft vary from $1.54 \%-2.58 \%{ }^{21}$ Mohamed $\mathrm{A}$ et al. ${ }^{22}$ observed that incidence of bone debris after ACL reconstruction surgery was $15 \%$ if thorough debridement was done and $65 \%$ if no specific debridement protocol was followed. ${ }^{22}$ Treatment of patellar tendon ossification is still not well established. However, some authors showed good results with surgical resection of ossification. ${ }^{14}$ We managed our patient non-operatively as he did not have any limitation of daily activity. In the present case report, we observed that patient had both patellar tendon ossification and heterotrophic ossification. There was no case reported in the literature which showed both patellar tendon ossification and heterotrophic calcification in the same patient after ACL reconstruction with BPTB graft.

Table I Review of literature

\begin{tabular}{lllll}
\hline Article & Age/sex & $\begin{array}{l}\text { Type } \\
\text { of } \\
\text { graft }\end{array}$ & Treatment & $\begin{array}{l}\text { Duration } \\
\text { of injury }\end{array}$ \\
\hline $\begin{array}{l}\text { Gianluca } \\
\text { Camillieri }\end{array}$ & $42 /$ male & BPTB & $\begin{array}{l}\text { Excision of } \\
\text { ossification }\end{array}$ & - \\
Homero Valencia & $29 /$ male & BPTB & $\begin{array}{l}\text { Excision of } \\
\text { ossification }\end{array}$ & $\begin{array}{l}10 \\
\text { months }\end{array}$ \\
$\begin{array}{l}\text { Mehmet Erdil } \\
\text { Fahri Erdogan }\end{array}$ & $36 /$ male & BPTB & $\begin{array}{l}\text { Excision of } \\
\text { ossification }\end{array}$ & 3 months \\
\hline
\end{tabular}

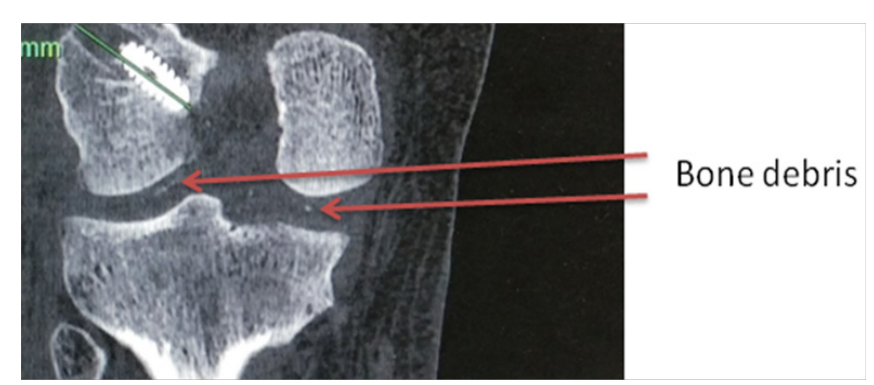

Figure 2 Post-operative (5th day) CT scan showing loose bone debris in the joint.

\section{Acknowledgements}

Written and informed consent was taken from the patient.

\section{Conflict of interest}

No conflict of interest.

\section{References}

1. Heijne A, Werner S. A 2-year follow-up of rehabilitation after ACL reconstruction using patellar tendon or hamstring tendon grafts: a prospective randomised outcome study. Knee Surg Sports Traumatol Arthrosc. 2010;18(6):805-13.

2. Li S, Chen $Y$, Lin Z, et al. A systematic review of randomized controlled clinical trials comparing hamstring autografts versus bone-patellar tendonbone autografts for the reconstruction of the anterior cruciate ligament. Arch Orthop Trauma Surg. 2012;132(9):1287-97.

3. Nwachukwu BU, Voleti PB, Berkanish P, et al. Return to Play and Patient Satisfaction After ACL Reconstruction: Study with Minimum 2-Year Follow-up. J Bone Joint Surg Am. 2017;99(9):720-5.

4. Gudas R, Jurkonis R, Smailys A. Comparison of Return to Pre-Injury Sport After $10 \mathrm{~mm}$ Size Bone-Patellar Tendon-Bone (BPTB) versus $8 \mathrm{~mm}$ Hamstring Anterior Cruciate Ligament Reconstruction: A Retrospective Study with a Two-Year Follow-Up. Med Sci Monit. 2018;24:987-96.

5. Monaghan PKL, Salem H, Ross KE, et al. Long-term outcomes in anterior cruciate ligament reconstruction: a systematic review of patellar tendon versus hamstring autografts. Orthop J Sports Med. 2017;5(6).

6. Kartus J, Movin T, Karlsson J. Donor-site morbidity and anterior knee problems after anterior cruciate ligament reconstruction using autografts. Arthroscopy. 2001;17(9):971-80.

7. Webster KE, Feller JA, Hartnett N, et al. Comparison of patellar tendon and hamstring tendon anterior cruciate ligament reconstruction: a 15year follow-up of a randomized controlled trial. Am J Sports Med. 2016;44(1):83-90.

8. Matsumoto H, Kawakubo M, Otani T, et al. Extensive post-traumatic ossification of the patellar tendon: a report of two cases. J Bone Joint Surg Br. 1999;81(1):34-6.

9. Majeed H, McBride D. Massive Patellar Tendon Ossification: Excision and Simultaneous Total Knee Replacement. Scientific Pages Orthop Surg. 2017;1(1):17-9.

10. Kelly MA, Insall JN. Postpatellectomy Extensive Ossification of Patellar Tendon: A Case Report. Clin Orthop Relat Res. 1987;(215):148-52.

11. Gosselin RA, Belzer JP. Contreras DM. Heterotopic ossification of the patellar tendon following intramedullary nailing of the tibia: report on two cases. J Trauma. 1993;34(1):161-3.

12. Tan L, Wang T, Li Y-H, et al. Patellar tendon ossification after retrograde intramedullary nailing for distal femoral shaft fracture: A case report and review of the literature. Medicine. 2017;96(47):e8875.

13. Yamamoto N, Miki T, Nishiyama A, et al. Extensive Bilateral Patellar Tendon Ossification in a Spinal Cord Injury Patient. Acta Med Okayama. 2018;72(1):89-93.

14. Valencia H, Gavín C. Infrapatellar heterotopic ossification after anterior cruciate ligament reconstruction. Knee Surg Sports Traumatol Arthrosc. 2007;15(1):39-42.

15. Erdil M, Aşı M, Sen C, et al. Heterotopic bone formation following anterior cruciate ligament reconstruction with BPTB autograft. Acta Orthop Traumatol Turc. 2012;46(1):72-6.

16. Camillieri G, Sanzo V, Ferretti M, et al. Patellar tendon ossification after anterior cruciate ligament reconstruction using bone-patellar tendonbone autograft. BMC Musculoskelet Disord. 2013;14:164. 
17. Erdogan F, Aydingoz O, Kesmezacar H, et al. Calcification of the patellar tendon after ACL reconstruction. Knee Surg Sports Traumatol Arthrosc. 2004;12(4):277-9.

18. Lerat JL, Besse JL, Vincent P, et al. Sequelae in the knee extensor system following graft removal for the "Mac in Jones" type procedure. Rev Chir Orthop Reparatrice Appar Mot. 1995;81(5):404-9.

19. Erdogan F, Aydingoz O, Kesmezacar H, et al. Calcification of the patellar tendon after ACL reconstruction. A case report with long-term follow-up. Knee Surg Sports Traumatol Arthrosc. 2004;12(4):277-9.
20. Bonamo JJ, Krinick RM, Sporn AA. Rupture of the patellar ligament after use of its central third for anterior cruciate reconstruction. A report of two cases. J Bone Joint Surg Am. 1984;66(8):1294-7.

21. Bhandary B, Shetty S, Bangera VV. To study the incidence of heterotopic ossification after anterior cruciate ligament reconstruction. J Clin Diagn Res. 2013;7(5):888-91.

22. Imam MA, Abdelkafy A, Dinah F, et al. Does bone debris in anterior cruciate ligament reconstruction really matter? A cohort study of a protocol for bone debris debridement. SICOT-J. 2015;1:4. 